Research Article

\title{
Childcare Correlates of Physical Activity, Sedentary Behavior, and Adiposity in Preschool Children: A Cross-Sectional Analysis of the SPLASHY Study
}

\author{
Amar Arhab (D, ${ }^{1}$ Nadine Messerli-Bürgy, ${ }^{1,2}$ Tanja H. Kakebeeke, ${ }^{3,4}$ Stefano Lanzi, \\ Kerstin Stülb, ${ }^{2}$ Annina E. Zysset, ${ }^{3}$ Claudia S. Leeger-Aschmann, ${ }^{5}$ Einat A. Schmutz, ${ }^{5}$ \\ Andrea H. Meyer, ${ }^{6}$ Simone Munsch, ${ }^{2}$ Susi Kriemler, ${ }^{5}$ Oskar G. Jenni, ${ }^{3,4}$ \\ and Jardena J. Puder ${ }^{1}$ \\ ${ }^{1}$ Obstetric Service, Lausanne University Hospital, Lausanne, Switzerland \\ ${ }^{2}$ Department of Clinical Psychology and Psychotherapy, University of Fribourg, Fribourg, Switzerland \\ ${ }^{3}$ Child Development Center, University Children's Hospital Zurich, Zurich, Switzerland \\ ${ }^{4}$ Children's Research Center, University Children's Hospital Zurich, Zurich, Switzerland \\ ${ }^{5}$ Epidemiology, Biostatistics and Prevention Institute, University of Zurich, Zurich, Switzerland \\ ${ }^{6}$ Department of Psychology, University of Basel, Basel, Switzerland
}

Correspondence should be addressed to Amar Arhab; amar.arhab@unil.ch

Received 27 April 2018; Revised 25 September 2018; Accepted 25 October 2018; Published 11 November 2018

Academic Editor: Linda M. Gerber

Copyright (C) 2018 Amar Arhab et al. This is an open access article distributed under the Creative Commons Attribution License, which permits unrestricted use, distribution, and reproduction in any medium, provided the original work is properly cited.

Background. The childcare (CC) environment can influence young children's physical activity (PA), sedentary behavior (SB), and adiposity. The aim of the study was to identify a broad range of CC correlates of PA, SB, and adiposity in a large sample of preschoolers. Methods. 476 preschool children (mean age 3.9 yrs; $47 \%$ girls) participated in the Swiss Preschoolers' Health Study (SPLASHY). PA and SB were measured by accelerometry. Outcome measures included total PA (TPA), moderate-to-vigorous PA (MVPA), SB, body mass index (BMI), and skinfold thickness (SF). PA measures consisted of both daily PA during CC attendance days and overall daily PA (CC and non-CC days). Results. We identified the following CC correlates for higher TPA and/or higher MVPA or lower SB during CC attendance days: older age, sex (boys), more frequent child-initiated interactions during CC, mixing different ages within a group, and the presence of a written PA policy in the CC (all $p \leq 0.02$ ). The CC correlates for overall TPA and/or MVPA or lower overall SB including both CC and non-CC days were the following: older age, sex (boys), more frequent child-initiated interactions during CC, mixing different ages within a group, less parental PA involvement in the CC, and having a larger surface area in CC (all $p \leq 0.046$ ). Correlates for lower SF were sex (boys) and parental PA involvement in the CC (all $p \leq 0.02)$, and, for lower BMI, only increased age $(p=0.001)$ was a correlate. Conclusions. More frequent child-initiated interactions and mixing different ages in CC, the presence of a written PA policy, and a larger CC surface are correlates of PA and SB during CC attendance days and/or of overall PA. Parental involvement in CC PA projects was a correlate for reduced body fat. These novel factors are mostly modifiable and can be tackled/addressed in future interventions.

\section{Introduction}

The recent World Health Organisation (WHO) report about childhood obesity prevention measures includes the promotion of physical activity (PA) and the reduction of sedentary behavior (SB) during the early years [1]. Since many young children spend a large portion of their week in childcare (CC) [2,3], the CC is an ideal context within which to target children's health behaviors. Indeed, research has shown that interventions in CC can have an impact on young children's activity behaviors and/or on their body weight $[3,4]$, but there is still a need for more data [5]. 
Therefore, it is important to expand our knowledge on CC correlates of young children's PA, SB, and body weight in order to tailor future interventions $[5,6]$.

Childcare correlates of PA and SB differ by country and CC [6-8], and most studies have focused on PA-specific correlates. Initial studies that identified CC correlates originated from the US showed that active opportunities, staff PA training and education, portable play equipment, and sufficient outdoor space were positively related to children's in-care PA behavior [9-11]. However, these US studies have not looked at correlates of SB. On the other hand, a Canadian study found that sedentary environment, sedentary opportunities, and fixed play equipment were correlates of in-care SB [12]. Three studies from Australia have identified written PA policy, staffled structured PA, staff joining in active play, less time spent indoor before going outdoor, use of indoor space for gross motor activities, lower child-staff ratios, presence of outdoor fixed equipment, and increased number of outdoor spaces with natural ground coverings as positive correlates of young children's in-care PA $[7,13,14]$. In regard to correlates of SB, lower child-staff ratio, using of indoor space for gross motor activities, and the presence of outdoor fixed equipment were identified as correlates of less in-care SB [7, 13]. In three European studies, other correlates such as active opportunities in CC, size of indoor area per child, and location of preschool building on the playground have been linked to in-care PA and/or SB in preschool children [15-17]. For example, data from the UK showed that only one correlate, e.g., active opportunities, was associated with children's in-care PA [16]. According to the authors, correlates of the CC environment may have a more limited influence on children's PA in countries such as the UK, where policies advocate child-driven play and moving freely indoors and outdoors [16].

As PA, SB, and obesity are related and are all important health outcomes of children's lives [18], it is important to include not only correlates of PA and SB but also of childhood adiposity. We are aware of one study that evaluated CC correlates of adiposity [19]. The authors found that less minutes spent in SB, more time spent in active play, less offers of high fat/high sugar foods, and less opportunities for sedentary activities during CC attendance days were associated with lower BMI or lower risk of being overweight. Clearly, additional studies investigating CC correlates of obesity in young children are needed.

When investigating CC correlates, the socioecological model provides a useful framework for conceptualizing potential correlates of children's behavior and health across 5 domains: demographic/biological, psychological/cognitive/ emotional, behavioral, sociocultural, and physical environment [20-22]. Such a framework will allow us to have a broader and comprehensive perspective across the whole spectrum of the ecological model to clearly identify all potential correlates of $\mathrm{PA}, \mathrm{SB}$, and obesity. For example, there is a lack of knowledge about CC correlates at the sociocultural level such as organisation of different age classes or policies of the CC [23]. Additionally, several correlates of PA, SB, and adiposity could also be important novel correlates within the CC setting: socioeconomic status (SES) of the CC [24-26], language region of the CC $[27,28]$, and psychological and behavioral correlates of the children while in CC, such as peer relationship problems and eating behavior [29-31].

The previously mentioned studies have all evaluated PA correlates by focusing on PA during CC attendance. To our knowledge, only one study examined the effect of the CC environment on children's PA levels over the entire day [32]. The authors found during one single day of observation that 60 minutes of outdoor time or active time during CC attendance was related to increased overall PA of the entire 24hour day. It could indicate a compensatory effect of CC correlates by either reducing or increasing PA at home. It's important to study if CC correlates also impact on overall daily PA and SB. Thus, it is important to include also days without CC attendance, as it's the overall daily PA behaviors that are related to many important health indicators [33].

The aim of the current study was to investigate CC correlates of (1) PA and SB during CC attendance days, (2) overall daily PA and SB including all days, both CC and nonCC days, and (3) adiposity using data of a large cross-sectional study of 2- to 6-year-old preschoolers. We specifically aimed to include a large range of correlates and integrate more novel correlates based on the socioecological model.

\section{Methods}

2.1. Study Sample and Design. The Swiss Preschoolers' Health Study (SPLASHY) is a multisite prospective cohort study including 476 preschool children aged 2 to 6 years within two sociocultural areas of Switzerland (German and French speaking parts) (ISRCTN41045021). Preschool children were recruited from 84 CC within five cantons/provinces of Switzerland (Aargau, Bern, Fribourg, Vaud, and Zurich), which together made up to $50 \%$ of the Swiss population in 2013. Recruitment lasted from March 2014 until December 2015. The selection procedure was stratified according to one stratum with 4 levels: urban community $(>100,000$ inhabitants or biggest city in the canton/province) and rural community, each with high socioeconomic status (SES) and low SES defined according to the median maternal educational level of the community $[34,35]$. For the larger urban communities, the list of CC centers was divided into high and low SES using the definition of SES according to the Swiss neighbourhood index of socioeconomic status [25]. The detailed study design and the overall objectives have been published previously [36]. The present analysis focuses on the baseline cross-sectional assessment. The study was approved by all local ethical committees (No. 338/13 for the Ethical Committee of the Canton/Province of Vaud as the main ethical committee). Parents provided written informed consent and children provided oral consent. Data collection was conducted in parallel at all study sites according to standardized procedures.

\subsection{Assessment of the Outcome Variables}

2.2.1. Physical Activity and Sedentary Behavior Assessment. Children's PA and SB were objectively monitored using an accelerometer (wGT3X-BT, ActiGraph, Pensacola, Florida, 
USA). Children were asked to continuously wear the accelerometer around the right hip for seven consecutive days, including at nights. The device was removed for water-based activities, e.g. showering or swimming. Children, parents, and CC staff received detailed instructions on the use of the activity monitor. PA data (raw data) were collected at a sampling frequency of $30 \mathrm{~Hz}$ and downloaded in 3-s epochs. For data analysis, periods of 20 minutes and more of consecutives zero readings were removed and considered as nonwear time [37]. All recordings between $9 \mathrm{pm}$ and $7 \mathrm{am}$ were excluded, as this most likely reflected the hours spent sleeping. Measurements with a minimum of 10 hours recordings were considered a valid day. Epoch length was set at 60 seconds to determine the level of total PA (TPA) as mean accelerometer counts per minute (cpm), moderate-tovigorous PA (MVPA; $\mathrm{min} /$ day), and SB (min/day). MVPA and SB were defined as $\geq 2120$ counts/60-s and $\leq 239$ counts/60-s, respectively, using Butte et al. cutoffs [38], developed specifically for young children using the same accelerometer model. The above age-specific (preschoolers) data collection and processing criteria were based on a recent review [39]. Physical activity measures included daily PA during CC attendance days (full-day attendance) and overall daily PA (including all days, both CC and non-CC days), the latter to evaluate the impact of the correlates on overall PA. For 394 (83\%) children, measurements across at least 3 days ( 2 weekdays and 1 weekend day) were available, whereas for the remaining $82(17 \%)$, only one valid measurement day was available. We therefore tested whether these two groups differed from each other with respect to mean values for any of the eight outcomes used in our study, but there were no significant differences $(p>0.05$ for any of the eight outcomes, using $t$-tests for independent samples). Therefore, the two groups were collapsed.

2.2.2. Adiposity Assessment. Two different adiposity measures (BMI and skinfold thickness) were assessed in the CC center by 5 different well-trained examiners (CL, AZ, KS, $\mathrm{AA}$, and $\mathrm{NM}$ ). Height was measured to the nearest $0.1 \mathrm{~cm}$ with a stadiometer and weight to the nearest $0.1 \mathrm{~kg}$ (Seca, Basel, Switzerland) using standardized procedures. BMI was then calculated as weight/height squared $\left(\mathrm{kg} / \mathrm{m}^{2}\right)$. All measurements were taken barefoot and in light clothing. Overweight and obesity were defined based on the WHO [40] criteria. Skinfold thickness (SF) was measured using standard procedures [41] in triplicate to the nearest $0.1 \mathrm{~mm}$ with Harpenden calipers (HSK-BI, British Indicators, UK) at the triceps, biceps, subscapular, and suprailiac crest. The sum of all four skinfolds was calculated and referred to as "skinfold thickness or body fat."

2.3. Childcare Correlates. The correlates of the CC environment (Table 1) were mainly assessed through a modified Nutrition and Physical Activity Self-Assessment for Child Care (NAP SACC) Questionnaire [42], completed by the main CC educator responsible for the group of the assessed children. We used the socioecological model of health behavior proposed by Sallis et al. [20] to conceptualize and classify potential correlates according to five domains: (1) demographic/biological, (2) psychological/cognitive/ emotional, (3) behavioral, (4) sociocultural, and (5) physical environment [20]. A detailed description of all 35 potential correlates is provided in Table 1. Nutritional correlates $(n=15)$ were only used for adiposity as outcome variable.

2.3.1. Demographic and Biological Domains. Five variables from the demographic/biological domain were included in the analysis. Age and sex were extracted from parental report. We also included the sociocultural language region of the CC, SES, and the rural-urban location of the community where the CC was located (see "Study sample and design" for more details).

\subsubsection{Psychological, Cognitive, and Emotional Domains.} Three variables from the psychological domain were included that assessed educators and children while in CC, as children's social and peer problems are known to be related to childhood obesity [30]. Thus, "child-initiated interaction" ("child goes toward peers") was examined using the item "good friend" of the "peer relationship problems" scale from the Strengths and Difficulties Questionnaire (SDQ) [43]. It assesses how easily the child goes towards peers. It is thus a child-initiated interaction. "Playing peers" was assessed in order to grasp the number of peers the child plays with during CC [44]. Both items were assessed by the main educator. We also investigated the support of the educators for children by rating the overall "staff support" using direct observation by the $\mathrm{PhD}$ and postdocs during the testing. For the latter, we looked at support of educators for children in finding solutions to problems. We thereby used a scale ranging from 1 to 5 with higher numbers signifying more support.

2.3.3. Behavioral Domain. Based on previous research, eating behavior was chosen to assess behavioral correlates $[1,30,31]$. We used three items from the Child Eating Behavior Questionnaire (CEBQ) to investigate the contribution of individual differences in eating behavior (food approach and food avoidant, while in CC) to the development of obesity [45]. Item selected for the "food approach" subscale was child's interest in food (enjoyment of food scale). Items selected for the "food avoidant" subscale were child eating when upset (emotional overeating scale) and child leaving food on the plate after meals (satiety responsiveness scale). The main educator in charge completed the questionnaire.

2.3.4. Sociocultural Domain. Twenty variables from the sociocultural domain were selected. Mixing different ages within a CC group was assessed via CC questionnaire and defined as the number of age classes within a CC group. Five PA items and 13 nutritional items from the NAP SACC were considered representing the global CC policies. For example, daily structured PA, written PA policy, and staff nutrition 
TABle 1: Potential childcare correlates of physical activity, sedentary behavior, and adiposity.

\begin{tabular}{|c|c|c|c|}
\hline Correlates & Source & Description & $\begin{array}{c}\text { Mean (SD) or } \\
\%\end{array}$ \\
\hline \multicolumn{4}{|l|}{ (1) Demographic-biological } \\
\hline Age (years) & Parental report & Child's age in years & $3.9(0.7)$ \\
\hline Sex (\% male $)$ & Parental report & Child's sex & 53 \\
\hline $\begin{array}{l}\text { Sociocultural region }(\% \\
\text { German) }\end{array}$ & Regional location of the CC & $\begin{array}{c}\text { German or French speaking region of } \\
\text { Switzerland }\end{array}$ & 74 \\
\hline CC SES (\% high) & Swiss neighbourhood index & $\begin{array}{l}\text { Socioeconomic status of the community of the } \\
\text { CC }\end{array}$ & 67 \\
\hline CC rural/urban (\% urban) & Location of the CC & $\begin{array}{l}\text { Urban defined as biggest cities of each canton } \\
\text { and cities }>100,000 \text { inhabitants }\end{array}$ & 60 \\
\hline
\end{tabular}

(2) Psychological-cognitive-

emotional

Staff support

Child-initiated interaction

Playing peers

(3) Behavioral

Eating behavior

Interest in food*

Eating when upset*

Leaving food on the plate*
Direct observation by research staff

SDQ questionnaire

Subscale: peer relationship problems

$\mathrm{CC}$ questionnaire
Staff support for children in finding solutions to problems: Scale 1-5 increasing with more support

Child goes toward other children: Likert's scale 0-4 increases with child going more toward other children

$N$ of peers the child plays with during CC
$4.2(0.7)$
CEBQ questionnaire Subscale: enjoyment of food CEBQ questionnaire Subscale: emotional overeating

CEBQ questionnaire Subscale: satiety responsiveness
(4) Sociocultural Organisational policies Mixing different ages $P A$ policies

Staff participation in PA

Staff PA training

Written PA policy (\% yes)

Daily PA (\%)

Daily structured PA (min)

Parental PA involvement (\% yes)

Nutritional policies

Staff nutrition training*

Staff food encouragement*

Children self-service*

Clean plate*
CC questionnaire

NAP SACC questionnaire Subscale: PA

NAP SACC questionnaire Subscale: PA

NAP SACC questionnaire Subscale: PA

NAP SACC questionnaire Subscale: PA

NAP SACC questionnaire Subscale: PA

CC questionnaire

NAP SACC questionnaire Subscale: nutrition

NAP SACC questionnaire Subscale: nutrition

NAP SACC questionnaire Subscale: nutrition

NAP SACC questionnaire Subscale: nutrition
Child's interest in food: Likert's scale 0-4 increases with child's interest in food

Child eating more when upset: Likert's scale $0-4$ increases with child eating more when upset Child leaving food on the plate after meals:

Likert's scale $0-4$ increases with child leaving food on plate
$N$ of different age classes within a CC group

$4.2(1.3)$

Staff participation during free PA: Likert's scale $0-3$ increases with more staff participation $N$ of staff members with PA training and education

Presence of a written policy for promoting PA

Time dedicated to daily PA per CC

$\begin{array}{cc}<30 \text { minutes } & 2 \\ 31-60 \text { minutes } & 25 \\ 61-90 \text { minutes } & 20 \\ >90 \text { minutes } & 53\end{array}$

Time in minutes dedicated to daily structured PA

Parental involvement in PA projects set up by the $\mathrm{CC}$

$N$ of staff members with children nutritional training and education

Staff encouraging children to health all kind of healthy foods: Likert's scale 1-4 increases with more encouragement from staff

Children serve themselves without any help from staff: Likert's scale 1-4 increases with more food self-service from children

Children are encouraged to finish their plate:

Likert's scale 1-4 increases with more encouragement from staff to finish their plate 
TABLE 1: Continued.

\begin{tabular}{|c|c|c|c|}
\hline Correlates & Source & Description & $\begin{array}{c}\text { Mean (SD) or } \\
\%\end{array}$ \\
\hline Fruits availability* & $\begin{array}{l}\text { NAP SACC questionnaire } \\
\text { Subscale: nutrition }\end{array}$ & $\begin{array}{l}\text { Frequency of fruits availability in CC: Likert's } \\
\text { scale 1-4 increases with more fruits availability }\end{array}$ & $3.8(0.4)$ \\
\hline Vegetables availability* & $\begin{array}{l}\text { NAP SACC questionnaire } \\
\text { Subscale: nutrition }\end{array}$ & $\begin{array}{c}\text { Frequency of vegetables availability in CC: } \\
\text { Likert's scale } 1-4 \text { increases with more vegetables } \\
\text { availability }\end{array}$ & $3.4(0.5)$ \\
\hline Sweet drinks availability* & $\begin{array}{l}\text { NAP SACC questionnaire } \\
\text { Subscale: nutrition }\end{array}$ & $\begin{array}{c}\text { Frequency of sweet drinks availability in CC: } \\
\text { Likert's scale } 0-3 \text { increases with more sweets } \\
\text { drinks availability }\end{array}$ & $0.1(0.4)$ \\
\hline Juices availability* & $\begin{array}{l}\text { NAP SACC questionnaire } \\
\text { Subscale: nutrition }\end{array}$ & $\begin{array}{l}\text { Frequency of juices availability in CC: Likert's } \\
\text { scale } 0-3 \text { increases with more juices availability }\end{array}$ & $0.7(1.1)$ \\
\hline Water availability* & $\begin{array}{l}\text { NAP SACC questionnaire } \\
\text { Subscale: Nutrition }\end{array}$ & $\begin{array}{c}\text { Availability of drinking water in CC: Likert's } \\
\text { scale } 0-3 \text { increases with more water being freely } \\
\text { available }\end{array}$ & $2.9(0.4)$ \\
\hline Using food as reward* & $\begin{array}{l}\text { NAP SACC questionnaire } \\
\text { Subscale: nutrition }\end{array}$ & $\begin{array}{l}\text { Food is used to reward desired behavior: Likert's } \\
\text { scale } 0-3 \text { increases with more food reward }\end{array}$ & $0.1(0.3)$ \\
\hline Using food as regulator* & $\begin{array}{l}\text { NAP SACC questionnaire } \\
\text { Subscale: nutrition }\end{array}$ & $\begin{array}{l}\text { Food is used to control behavior or as } \\
\text { punishment: Likert's scale } 0-3 \text { increases with } \\
\text { more often food used to control behavior }\end{array}$ & $0.1(0.3)$ \\
\hline \multicolumn{4}{|l|}{ (5) Physical environment } \\
\hline CC surface & CC questionnaire & $\begin{array}{c}\text { Ratio of the CC surface }\left(\mathrm{m}^{2}\right) \text { per number of } \\
\text { children }\end{array}$ & $7.0(5.1)$ \\
\hline \multicolumn{3}{|r|}{ 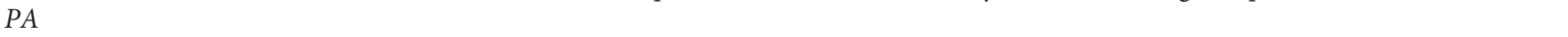 } & $2.8(1.2)$ \\
\hline PA indoor space (\% yes) & $\begin{array}{l}\text { NAP SACC questionnaire } \\
\text { Subscale: PA }\end{array}$ & Presence of indoor space dedicated for PA & 80 \\
\hline PA outdoor space (\% yes) & $\begin{array}{c}\text { NAP SACC questionnaire } \\
\text { Subscale: PA }\end{array}$ & Presence of outdoor space dedicated for PA & 91 \\
\hline Fixed PA equipment & $\begin{array}{l}\text { NAP SACC questionnaire } \\
\text { Subscale: PA }\end{array}$ & $N$ of fixed equipment related to $\mathrm{PA}$ & $2.9(2.5)$ \\
\hline Mobile PA equipment & $\begin{array}{c}\text { NAP SACC questionnaire } \\
\text { Subscale: PA }\end{array}$ & $\mathrm{N}$ of mobile equipment related to $\mathrm{PA}$ & $18.9(19.8)$ \\
\hline
\end{tabular}

$N=$ number $\mathrm{CC}=$ childcare; $\mathrm{SES}=$ socioeconomic status; $\mathrm{SDQ}=$ strength and difficulties questionnaire; $\mathrm{CEBQ}=$ child eating behavior questionnaire; $\mathrm{PA}=$ physical activity; NAP SACC $=$ nutrition and physical activity self-assessment for childcare; ${ }^{*}=$ correlates included in the models with adiposity as outcome. We used a modified version of the NAP SACC questionnaire.

training were included. Parental PA involvement in the CC was also assessed, as parental involvement is a key element in obesity prevention $[4,46]$.

2.3.5. Physical Environment Domain. Six variables from the physical environmental domain were selected. CC surface was assessed and represents the ratio of the CC surface $\left(\mathrm{m}^{2}\right)$ per number of children since classroom and playground size are important characteristics in promoting PA in CC settings [9]. Childcare attendance of the child was based on the parental report and represents the number of full days per week children attend the CC. In addition, four PA-related items from the NAP SACC questionnaire were included.

2.4. Statistical Analyses. In order to analyse the association between CC environment and children's PA, SB, and adiposity, we used regression model techniques. Children's PA and $\mathrm{SB}$ outcomes were as follows: total PA, moderate and vigorous $\mathrm{PA}$, and $\mathrm{SB}$ (all 3 both during CC attendance days and overall daily PA). Adiposity outcomes were as follows: BMI and SF. These were each analysed in a separate model.
Two different regression models were applied. First, we used a multiple regression model which included the entire list of putative correlates to enable comparison with previous studies. However, the intraclass correlation coefficient (ICC) between CC was close to 0 , and thus we did not use multilevel regression analyses. Because multiple regression models often suffer from overfitting leading to models having low predictive accuracy when predicting new samples [47], we also used a second model, i.e., a variable selection procedure, the least absolute shrinkage and selection operator (lasso) [48]. The lasso model is more biased but exhibits strongly increased predictive accuracy compared to multiple regression models. The lasso technique eliminates unimportant correlates from the model by setting their coefficients to zero while the relevant correlate variables remain in the model, their coefficients being usually shrunk towards zero. Note that the two coefficients for age and sex were deliberately not shrunk and thus always kept in the lasso model, as prior knowledge had demonstrated their influence on PA and adiposity $[20,23,49]$. Predictive accuracy of both the multiple regression model and the lasso were determined by ten-fold cross validation [50]. Performance measures 
reported were the variance explained $\left(R^{2}\right)$ and root mean square error (RMSE). For the lasso model, we set the tuning parameter alpha to 0.95 [51].

All outcomes and correlates were transformed if necessary to meet regression analysis assumptions (normality and homoscedasticity). For the lasso model, all variables were standardized prior to analysis. Since for the lasso no tests of significance are yet readily available, we refrain from reporting $p$ values $[52,53]$. To run the lasso model, the two $\mathrm{R}$ packages glmnet and caret were used [54, 55].

The data contained missing values. Overall, $14 \%$ of values were missing (between 0 and $35 \%$, depending on the variable), and $77 \%$ of all children and $84 \%$ of all variables had at least one missing value. Therefore, we used multiple imputation techniques to estimate missing values. To this end, prior to any analysis, missing values were repeatedly (i.e., fifty times) imputed using chained equations as implemented in the R package mice [56]. This resulted in fifty complete datasets in which missing values had been replaced by estimated values. Subsequent regression models were thus run fifty times using each of the complete datasets in turn, and results then pooled across the fifty datasets. Since to our knowledge no technique exists to date for combining lasso-based results from several data files, we determined the importance of each potential correlate by computing the mean and standard deviation of lasso coefficients across all fifty data files. In addition, we counted the number of times each putative correlate was contained in the best fitting lasso model according to cross validation (0-50). Note, however, that a value near 50 does not necessarily point to a correlate with high accuracy if the best fitting lasso model had a bad fit. Thus, mean standardized lasso coefficients give a better picture of the importance of a putative correlate. We added the lasso results in the appendix and only refer to major concordances and discordances in the text.

\section{Results}

The descriptive characteristics for all children are summarized in Table 2. Mean age was $3.9 \pm 0.7$ years and $47 \%$ of the participating children were girls. Participants provided an average of $5.6 \pm 0.9$ days of valid PA data, with a mean wearing time of 12.8 hours \pm 0.06 per day.

\subsection{Daily Physical Activity during Childcare Attendance Days} (Full-Day Attendance). Multivariate associations between the 20 potential correlates across all domains and TPA, MVPA, and SB during CC attendance days are presented in Table 3(a). Five variables from the demographic/biological, psychological/cognitive/emotional, and sociocultural domains were identified as correlates of PA: age (older), sex (boys), child-initiated interaction with other children during CC, mixing different ages within a CC group, and the presence of a written PA policy were positively associated with TPA and MVPA during CC attendance days (all $p \leq 0.02$ ). These five correlates accounted for $19 \%$ and $22 \%$ of the variance of time spent in TPA and MVPA, respectively. Three of 20 tested correlates from the psychological/cognitive/emotional and sociocultural domains were inversely related to SB during CC attendance days: child-initiated interaction with other children during CC, mixing different ages within a $\mathrm{CC}$ group, and the presence of a written PA policy were associated with less time spent in SB (all $p \leq 0.04)$. These three correlates accounted for $17 \%$ of the variance variability of the time spent in SB.

3.2. Overall Daily Physical Activity (during All Days, Childcare, and Nonchildcare Days). Associations between potential correlates across all domains and TPA, MVPA, and SB for overall PA are presented in Table 3(b). Four variables from the demographic/biological, psychological/ cognitive/emotional, and sociocultural domains were identified as correlates of overall PA: age (older), sex (boys), child-initiated interaction with other children during CC, and mixing different ages within a CC group were positively associated with TPA and MVPA (all $p \leq 0.045$ ). These four correlates accounted for $20 \%$ of the variance of the time spent in TPA. For MVPA, CC surface size from the physical environment domain was additionally identified as a correlate $(p=0.047)$. Age (older), sex (boys), child-initiated interaction with other children during CC, mixing different ages within a CC group, and CC surface size accounted for $27 \%$ of the variance of time spent in MVPA. For overall SB, two correlates from the demographic/biological and psychological/cognitive/emotional domains were associated with less SB: sex (boys) and childinitiated interaction with other children during CC $(p \leq 0.04)$. One correlate from the sociocultural domain was associated with more SB: parental involvement in PA projects of the CC $(p=0.03)$. Parental involvement in PA projects of the CC accounted for $14 \%$ of the variance of time spent in SB.

3.2.1. Adiposity. Multivariate associations between the 35 potential correlates (including nutritional variables) with BMI and skinfold thickness are presented in Table 4. For BMI, only age was inversely associated with BMI $(p=0.002)$. Age accounted for $11 \%$ of the variance of children's BMI. For skinfold thickness, sex (boys) and increased parental involvement in PA projects of the CC were associated with lower skinfold thickness $(p \leq 0.02)$. Sex (boys) and parental involvement in PA projects of the CC accounted for $18 \%$ of the variance of children's skinfold thickness.

3.2.2. Lasso Model. Lasso regression analysis was used to test robustness (accuracy and interpretability) of our results. Details are provided in Additional file 1. In summary, the standardized coefficient (SC) cutoff was, arbitrary, set at 0.07. Lasso results were very similar to the regression analysis for most of the correlates identified for TPA, MVPA, SB, BMI, and SF, corroborating the importance of these correlates mentioned in the current study. However, few discrepancies between regression and lasso were identified: child interaction with others in CC was associated 
TABLE 2: Descriptive characteristics of the participants.

\begin{tabular}{|c|c|}
\hline Characteristics & Total population $(n=476)$ \\
\hline Age (years) & $3.9 \pm 0.7$ \\
\hline \multicolumn{2}{|l|}{ Sex } \\
\hline Boys, $n(\%)$ & $246(53)$ \\
\hline Girls, $n(\%)$ & $217(47)$ \\
\hline \multicolumn{2}{|l|}{ Sociocultural region } \\
\hline German speaking, $n(\%)$ & $342(74)$ \\
\hline French speaking, $n(\%)$ & $121(26)$ \\
\hline Full-day attendance at CC & $2.8(1.2)$ \\
\hline \multicolumn{2}{|l|}{ Adiposity } \\
\hline Body mass index $\left(\mathrm{kg} / \mathrm{m}^{2}\right)$ & $16.02 \pm 1.35$ \\
\hline Skinfold thickness (mm) & $25.93 \pm 5.53$ \\
\hline \multicolumn{2}{|l|}{ Weight status* } \\
\hline Overweight, $n(\%)$ & $82(18)$ \\
\hline Obese, $n(\%)$ & $24(5)$ \\
\hline \multicolumn{2}{|c|}{ Daily physical activity during CC attendance days } \\
\hline TPA $(\mathrm{cpm})$ & $624 \pm 166$ \\
\hline $\operatorname{MVPA}(\mathrm{min} / \mathrm{d})$ & $46 \pm 58$ \\
\hline $\mathrm{SB}(\mathrm{min} / \mathrm{d})$ & $357 \pm 65$ \\
\hline \multicolumn{2}{|c|}{ Overall daily physical activity (CC and non-CC days) } \\
\hline TPA (cpm) & \\
\hline $\operatorname{MVPA}(\mathrm{min} / \mathrm{d})$ & $46 \pm 23$ \\
\hline $\mathrm{SB}(\mathrm{min} / \mathrm{d})$ & $366 \pm 56$ \\
\hline
\end{tabular}

$\mathrm{CC}=$ childcare; TPA = total physical activity; LPA = light physical activity; MPA = moderate physical activity; MVPA = moderate-to-vigorous physical activity; VPA = vigorous physical activity; $\mathrm{SB}=$ sedentary behavior; $\mathrm{cpm}=$ counts per minute; $\mathrm{min} / \mathrm{d}=$ minutes per day. Daily physical activity during CC attendance days denotes the physical activity and days where the child attends CC during the full day. Overall daily physical activity denotes physical activity during all days, i.e., CC and non-CC days. ${ }^{*}$ Based on the WHO criteria [40]. Cutoff for the physical activity intensities are based on Butte [38].

with overall TPA and MVPA in the regression model but was not significant in the lasso model $(\mathrm{SC}=0.06$ and 0.03 , respectively). Also, parental $\mathrm{PA}$ involvement in $\mathrm{PA}$ projects of the CC was associated with lower skinfold thickness in the regression model, but it was not significant in the lasso model $(\mathrm{SC}=-0.03)$. On the other side, some correlates were significant in the lasso model but not in the regression model: sex (boys), sociocultural region (German), higher SES of the CC, and larger CC surface were associated with less time spent in SB during $\mathrm{CC}$ attendance days $(\mathrm{SC}=0.10$, $-0.10,0.07$, and -0.08 , respectively). Additionally, sociocultural region (German) and larger CC surface were also shown to be associated with less time spent in overall SB in the lasso model ( $\mathrm{SC}=0.13$ and 0.08 , respectively). Finally, age was significant for skinfold thickness in the lasso model $(\mathrm{SC}=-0.07)$.

\section{Discussion}

Based on the socioecological model of health behavior, this study aimed at identifying a broad range of potential $\mathrm{CC}$ correlates of PA, SB, and adiposity in a large sample of preschool children using a cross-sectional design. Those correlates tested were selected based on the following 5 domains: demographic/biological, psychological/cognitive/ emotional, behavioral, sociocultural, and physical environment [20]. Thus, by using a socioecological model and taking into account a larger spectrum of CC correlates, we could identify novel and mostly modifiable correlates of daily PA and SB during CC days and/or of overall daily PA, such as increased child-initiated interactions with other children during CC, mixing different ages within a CC group, the presence of a written PA policy, and a larger CC surface. Parental involvement in PA projects of the CC was a correlate of reduced body fat.

4.1. Daily Physical Activity during Childcare Attendance Days. We measured PA during CC attendance days (full-day attendance), which is comparable to most other studies that measured PA during CC. In line with previous studies, we found that sex (boys) and age (older children) were consistent correlates of increased PA in preschool children $[7,17,23,57-60]$. This compelling evidence suggests that gender-specific strategies could be helpful when arranging the CC environment to promote PA. For example, providing more intentional opportunities for girls to initiate activities might increase their motivation and involvement in PA. Another potential strategy to increase young girls PA during CC might be to give more emphasis to teamoriented activities which consequently might increase girl's enjoyment of PA.

Using the socioecological model proposed by Sallis et al. [20], the present study identified three previously unmeasured CC correlates of preschoolers' PA and SB. These included child-initiated interactions with other children during CC, mixing different ages within a CC group, and the presence of a written PA policy. Indeed, children interacting with each other boosts child-initiated activities which have been associated with higher PA levels [11]. In line with the theory of observational learning, younger children may look up to older children and mimic 
TABLE 3: Associations of childcare correlates with physical activity and sedentary behavior.

\begin{tabular}{|c|c|c|c|c|}
\hline Outcomes & Correlates & $\beta$-coefficient & $95 \% \mathrm{CI}$ & $p$ value \\
\hline \multicolumn{5}{|c|}{ (a) Daily PA during childcare attendance days (full-day attendance) } \\
\hline \multirow{5}{*}{ Total PA (cpm) } & Age (years) & 45.14 & $(19.35,70.94)$ & 0.001 \\
\hline & Sex & -52.96 & $(-84.65,-21.26)$ & 0.001 \\
\hline & Child-initiated interaction* & 27.68 & $(4.67,50.69)$ & 0.02 \\
\hline & Mixing ages within a CC group & 26.61 & $(7.02,46.21)$ & 0.01 \\
\hline & Written PA policy & 53.28 & $(12.58,93.98)$ & 0.01 \\
\hline \multirow{5}{*}{ Moderate and vigorous $\mathrm{PA}(\mathrm{min} / \mathrm{d})$} & Age (years) & 10.09 & $(6.28,13.90)$ & 0.001 \\
\hline & Sex & -10.00 & $(-14.90,-5.11)$ & 0.001 \\
\hline & Child-initiated interaction* & 4.45 & $(1.11,7.79)$ & 0.01 \\
\hline & Mixing ages within a CC group & 3.74 & $(0.80,6.67)$ & 0.01 \\
\hline & Written PA policy & 7.14 & $(1.40,12.88)$ & 0.01 \\
\hline \multirow{3}{*}{ Sedentary time $(\mathrm{min} / \mathrm{d})$} & Child-initiated interaction* & -10.01 & $(-19.68,-0.33)$ & 0.043 \\
\hline & Mixing ages within a CC group & -10.35 & $(-18.09,-2.61)$ & 0.01 \\
\hline & Written PA policy & -23.92 & $(-39.99,-7.86)$ & 0.004 \\
\hline \multicolumn{5}{|c|}{ (b) Overall daily PA (childcare and nonchildcare days) } \\
\hline \multirow{4}{*}{ Total PA (cpm) } & Age (years) & 54.76 & $(33.23,76.29)$ & 0.001 \\
\hline & Sex & -58.97 & $(-86.64,-31.29)$ & 0.001 \\
\hline & Child-initiated interaction* & 20.81 & $(0.79,40.84)$ & 0.04 \\
\hline & Mixing ages within a CC group & 18.45 & $(1.07,35.84)$ & 0.04 \\
\hline \multirow{5}{*}{ Moderate and vigorous PA (min/d) } & Age (years) & 10.33 & $(7.24,13.42)$ & 0.001 \\
\hline & Sex & -11.39 & $(-15.41,-7.37)$ & 0.001 \\
\hline & Child-initiated interaction* & 3.55 & $(0.65,6.46)$ & 0.02 \\
\hline & Mixing ages within a CC group & 3.25 & $(0.71,5.78)$ & 0.01 \\
\hline & CC surface & 0.51 & $(0.01,1.01)$ & 0.047 \\
\hline \multirow{3}{*}{ Sedentary time $(\mathrm{min} / \mathrm{d})$} & Sex & 10.83 & $(0.24,21.41)$ & 0.045 \\
\hline & Child-initiated interaction* & -8.95 & $(-16.76,-1.13)$ & 0.03 \\
\hline & Parental PA involvement & 14.66 & $(1.61,27.71)$ & 0.03 \\
\hline
\end{tabular}

$\mathrm{PA}=$ physical activity; $\mathrm{CI}=$ confidence interval; $\mathrm{CC}=$ childcare; $\mathrm{cpm}=$ counts per minutes; $\mathrm{min} / \mathrm{d}=$ minutes per day; ${ }^{5}$-point Likert scale; Sex defines girls vs boys. Child interaction indicates child's tendency to go towards other peers while in CC. Mixing ages refers to the number of age classes in one group. Written PA policy refers to having a written PA policy promoting PA participation within the CC. Mobile equipment is expressed per 10 more equipment such as balls and frisbees. All outcome and predictor variables are unstandardized. The model always included age and sex. Daily physical activity during CC attendance days denotes the physical activity and days where the child attends CC during the full day. Overall daily physical activity denotes physical activity during all days, i.e., $\mathrm{CC}$ and non-CC days.

TABLE 4: Associations of childcare correlates with adiposity.

\begin{tabular}{|c|c|c|c|c|}
\hline Outcomes & Correlates & $\beta$-coefficient & $95 \% \mathrm{CI}$ & $p$ value \\
\hline BMI & Age (years) & -0.32 & $(-0.53,-0.11)$ & 0.002 \\
\hline \multirow{2}{*}{ Skinfold thickness } & Sex & 2.87 & $(1.81,3.94)$ & 0.001 \\
\hline & Parental PA involvement & -1.64 & $(-3.01,-0.28)$ & 0.02 \\
\hline
\end{tabular}

$\mathrm{PA}=$ physical activity; $\mathrm{CI}=$ confidence interval; $\mathrm{CC}=$ childcare; $\mathrm{BMI}=$ body mass index. Sex defines girls vs boys.

their behaviors [61]. Therefore, grouping older and younger children may encourage younger children to engage more in PA behaviors if the older children show the desired behavior. Finally, having a written policy defining the objectives, means, or strategies to promote PA is essential in elevating the importance of PA in a CC. Such policy can provide a conceptual framework and institutional commitment that would support participation, enjoyment, and safety in PA for preschoolers [62]. Interestingly, in the current sociocultural and physical CC environments in Switzerland, generic "activities" such as promoting child interactions and mixing ages within a CC group were stronger correlates of $\mathrm{PA}$ and $\mathrm{SB}$ than more "traditional" and PA-specific correlates. Based on these results, we would encourage spontaneous interaction between children of different age groups, in accordance with a previous study from the UK [16]. For example, encouraging free play activities where children cheer, imitate, and cooperate with each other will potentially boost their PA behaviors.

Contrary to findings in the current study, previous studies, mostly performed in the US, have found correlates such as staff training and education, structured PA, time devoted to indoor and outdoor PA opportunities, the presence of indoor and outdoor play spaces, portable equipment, and outdoor fixed equipment were associated with PA during CC attendance $[7,9,10,14,16,19,63]$. In our study, mobile equipment was borderline significant. The abovementioned differences could be attributable to study methodologies (choice of correlates, categorization and conceptualization of domains, and choice of observation instrument used to capture CC correlates), data acquisition 
of PA measures, and/or different sociocultural and political environments between countries.

4.2. Overall Daily Physical Activity (Childcare and Nonchildcare Days). Previous studies looking at CC correlates focused on PA during CC attendance. In addition to this, we were also interested in investigating the CC correlates that are related to overall PA including all days, as it is this overall PA that is important for overall health outcomes [33]. The current study found that child-initiated interactions with other children during CC, mixing different ages within a CC group, and the presence of a larger CC surface were related to a higher overall daily PA and/or more time spent in overall MVPA (CC and non-CC days). Additionally, parental involvement in PA projects of the CC was related to higher overall daily SB. The latter could hint toward a compensatory response behavior outside the CC environment. To our knowledge, only one previous study investigated the effects of the CC characteristics on PA over the 24-hour day during one day of observation [32]. In contrast to our results, the authors found that time provided outdoor and total active time were associated with higher MVPA. Similar to our data, they did not find that staff PA training, presence of indoor play space, and portable or fixed play equipment were correlates of children's MVPA. Thus, strategies in the CC promoting positive PA behaviors do not seem to lead to a complete compensation outside of the $\mathrm{CC}$ by reducing PA at home and might potentially even transfer outside the CC environment.

4.2.1. Adiposity. It was previously shown that obesogenic features of the CC environment have a substantial influence on obesity development in young children [19]. In our study, we found that age (older) was associated with lower BMI. We also found that sex (boys) and parental involvement in PA projects of the CC were associated with reduced body fat. Because boys are more engaged in higher PA intensities than girls, these specific intensities can offer protection against the development of obesity. Indeed, vigorous PA is strongly associated with lower adiposity in preschoolers [64].

The importance of parental involvement to change children's lifestyle has been reported in previous successful family-based interventions $[65,66]$. In our study, parental involvement in PA projects of the CC was associated with more overall SB and lower body fat. The reduced overall SB might be a compensatory mechanism, but it is not clearly understood and should be investigated in other studies. Regarding body fat, it could suggest that parental involvement might indirectly lead to favourable body composition since PA has a direct relationship with body fat and health behaviors [67]. On the other side, parental involvement in PA projects of the CC could just be a marker of increased overall parental commitment. In contrast to existing CC intervention studies, we only know of one study, done in the US, that looked at several CC correlates of adiposity [19]. The authors showed that less opportunities for sedentary activities, less minutes spent in SB, more time spent in active play, and less offers of high fat/high sugar foods in CC were all associated with lower BMI or lower risk of being overweight. Interestingly, they showed that $75 \%$ of the children used the computer on their observation day, while in the current study, no computer was used by the children in the CCs (data not shown). Thus, large sociocultural discrepancies between countries may impact on differences in the results. The observed differences between the current study and the previous one could also be attributable to the choice in the observation instrument for the correlates and in the PA measurements (wristwatch). Nonetheless, offering children opportunities to be active and involving parents in PA projects might encourage PA participation and/or protect against adiposity. For instance, informing parents about in-care PA behaviors of their child would improve communication between staff and parents, and eventually encourage parents to engage in the PA projects the CC.

Strengths of the current study include the conceptualization of potential correlates of PA, SB, and adiposity across 5 domains of the socioecological model of health behavior. Other strengths comprise the inclusion of novel correlates, multimethod approach including direct measurements, educator and parent-report information, objectively measured PA and SB both on CC attendance days and during CC and nonCC stay, and the relatively large study sample. Also, analyses applied of two statistical models, one of them novel, showed very similar results and gave a better picture on the importance of putative correlates and the robustness of our results. Limitations include the cross-sectional design which limits causation. Also, we did not perform a direct observation of the $\mathrm{CC}$ environment. However, the main responsible educator of the CC group shared the information regarding the CC correlates over several weeks of observation as opposed to an observation that lasts usually one day. This might especially be important for correlates such as daily PA and time spent outside that might fluctuate between days and according to the weather and season. The high number of correlates tested might have caused random findings. However, the lasso model through the penalized regression limited irrelevant correlates to be included in the model. Physical activity measurement during days with full-day CC attendance was not exactly time stamped to capture PA exactly during CC time only, which may in turn resulted in misclassification PA during CC. Also, two items from the psychological/cognitive/emotional domain ("playing peers" and "staff support") that were developed to meet the needs of our study were not validated in young children.

\section{Conclusions}

In conclusion, our findings provide evidence for novel CC correlates of preschoolers PA, SB, and adiposity using a socioecological model. Enabling spontaneous PA interactions between children of different age groups, establishing clear written PA policies, and a larger collaboration with parents in PA projects of the CC might lead to increased PA and reduced body fat in children. Integrating these 
mostly modifiable factors might be beneficial when planning future interventions.

\section{Data Availability}

The dataset supporting the conclusions of this article is available upon reasonable request to the corresponding author.

\section{Disclosure}

The funding bodies have no role in the design of the study and collection, analysis, and interpretation of data and in writing the manuscript.

\section{Conflicts of Interest}

The authors declare that they have no conflicts of interest.

\section{Authors' Contributions}

Simone Munsch, Susi Kriemler, Oskar G. Jenni, and Jardena J. Puder share last authorship.

\section{Acknowledgments}

We would like to thank all children, families, and day care centers that contributed data to SPLASHY. We also thank all students and research team for their valuable contribution. The study was funded through a Sinergia grant from the SNF (Grant no. CRSII3_147673) (http://p3.snf.ch/project147673) and by the Jacobs Foundation.

\section{Supplementary Materials}

Table 5: associations of childcare correlates with physical activity and sedentary behavior using lasso. Table 6: associations of childcare correlates with body composition using lasso. (Supplementary Materials)

\section{References}

[1] WHO, "Report of the commission on ending childhood obesity," January 2016, http://www.who.int/end-childhoodobesity/en/.

[2] J. J. Reilly, "Low levels of objectively measured physical activity in preschoolers in child care," Medicine \& Science in Sports \& Exercise, vol. 42, no. 3, pp. 502-507, 2010.

[3] G. S. Goldfield, A. L. Harvey, K. P. Grattan et al., "Effects of child care intervention on physical activity and body composition," American Journal of Preventive Medicine, vol. 51, no. 2, pp. 225-231, 2016.

[4] A. Bonvin, J. Barral, T. H. Kakebeeke et al., "Effect of a governmentally-led physical activity program on motor skills in young children attending child care centers: a cluster randomized controlled trial," International Journal of Behavioral Nutrition and Physical Activity, vol. 10, no. 1, p. 90, 2013.

[5] D. D. Bingham, S. Costa, T. Hinkley, K. A. Shire, S. A. Clemes, and S. E. Barber, "Physical activity during the early years a systematic review of correlates and determinants," American
Journal of Preventive Medicine, vol. 51, no. 3, pp. 384-402, 2016.

[6] S. G. Trost, D. S. Ward, and M. Senso, "Effects of child care policy and environment on physical activity," Medicine \& Science in Sports \& Exercise, vol. 42, no. 3, pp. 520-525, 2010.

[7] T. Hinkley, J. Salmon, D. Crawford, A. D. Okely, and K. D. Hesketh, "Preschool and childcare center characteristics associated with children's physical activity during care hours: an observational study," International Journal of Behavioral Nutrition and Physical Activity, vol. 13, no. 1, p. 117, 2016.

[8] R. R. Pate, K. A. Pfeiffer, S. G. Trost, P. Ziegler, and M. Dowda, "Physical activity among children attending preschools," Pediatrics, vol. 114, no. 5, pp. 1258-1263, 2004.

[9] M. Dowda, W. H. Brown, K. L. McIver et al., "Policies and characteristics of the preschool environment and physical activity of young children," Pediatrics, vol. 123, no. 2, pp. e261-e266, 2009.

[10] J. K. Bower, D. P. Hales, D. F. Tate, D. A. Rubin, S. E. Benjamin, and D. S. Ward, "The childcare environment and children's physical activity," American Journal of Preventive Medicine, vol. 34, no. 1, pp. 23-29, 2008.

[11] W. H. Brown, K. A. Pfeiffer, K. McIver, M. Dowda, C. L. Addy, and R. Pate, "Social and environmental factors associated with preschoolers' nonsedentray physical activity," Child Development, vol. 80, no. 1, pp. 45-58, 2009.

[12] P. Tucker, L. M. Vanderloo, S. M. Burke, J. D. Irwin, and A. M. Johnson, "Prevalence and influences of preschoolers' sedentary behaviors in early learning centers: a cross-sectional study," BMC Pediatrics, vol. 15, no. 1, p. 128, 2015.

[13] T. Sugiyama, A. D. Okely, J. M. Masters, and G. T. Moore, "Attributes of child care centers and outdoor play areas associated with preschoolers' physical activity and sedentary behavior," Environment and Behavior, vol. 44, no. 3, pp. 334-349, 2012.

[14] A. C. Bell, M. Finch, L. Wolfenden et al., "Child physical activity levels and associations with modifiable characteristics in centre-based childcare," Australian and New Zealand Journal of Public Health, vol. 39, no. 3, pp. 232-236, 2015.

[15] K. R. Hesketh, S. J. Griffin, and E. M. van Sluijs, "UK Preschool-aged children's physical activity levels in childcare and at home: a cross-sectional exploration," International Journal of Behavioral Nutrition and Physical Activity, vol. 12, no. 1, p. 123, 2015.

[16] K. R. Hesketh and E. M. van Sluijs, "Features of the UK childcare environment and associations with preschooler's incare physical activity," Preventive Medicine Reports, vol. 3, pp. 53-57, 2016.

[17] L. G. Olesen, P. L. Kristensen, L. Korsholm, and K. Froberg, "Physical activity in children attending preschools," Pediatrics, vol. 132, no. 5, pp. e1310-e1318, 2013.

[18] J. J. Reilly, "Physical activity, sedentary behaviour and energy balance in the preschool child: opportunities for early obesity prevention," Proceedings of the Nutrition Society, vol. 67, no. 3, pp. 317-325, 2008.

[19] S. B. Sisson, J. Li, J. A. Stoner et al., "Obesogenic environments in tribally-affiliated childcare centers and corresponding obesity rates in preschool children," Preventive Medicine Reports, vol. 3, pp. 151-158, 2016.

[20] J. F. Sallis, J. J. Prochaska, and W. C. Taylor, "A review of correlates of physical activity of children and adolescents," Medicine \& Science in Sports \& Exercise, vol. 32, pp. 963-975, 2000. 
[21] K. Glanz, B. Rimer, and K. Viswanath, Health Behavior and Health Education: Theory, Research, and Practice, Jossey-Bass, San Francisco, CA, USA, 2008.

[22] K. R. McLeroy, D. Bibeau, A. Steckler, and K. Glanz, "An ecological perspective on health promotion programs," Health Education Quarterly, vol. 15, no. 4, pp. 351-377, 1988.

[23] K. L. Tonge, R. A. Jones, and A. D. Okely, "Correlates of children's objectively measured physical activity and sedentary behavior in early childhood education and care services: a systematic review," Preventive Medicine, vol. 89, pp. 129139,2016

[24] R. Burgi, L. Tomatis, K. Murer, and E. D. de Bruin, "Spatial physical activity patterns among primary school children living in neighbourhoods of varying socioeconomic status: a cross-sectional study using accelerometry and Global Positioning System," BMC Public Health, vol. 16, no. 1, p. 282, 2016.

[25] R. Panczak, B. Galobardes, M. Voorpostel et al., "A Swiss neighbourhood index of socioeconomic position: development and association with mortality," Journal of Epidemiology and Community Health, vol. 66, no. 12, pp. 1129-1136, 2012.

[26] M. Sharifi, T. D. Sequist, S. L. Rifas-Shiman et al., "The role of neighborhood characteristics and the built environment in understanding racial/ethnic disparities in childhood obesity," Preventive Medicine, vol. 91, pp. 103-109, 2016.

[27] B. Bringolf-Isler, U. Mader, A. Dossegger et al., "Regional differences of physical activity and sedentary behaviour in Swiss children are not explained by socio-demographics or the built environment," International Journal of Public Health, vol. 60, no. 3, pp. 291-300, 2015.

[28] F. Burgi, U. Meyer, I. Niederer et al., "Socio-cultural determinants of adiposity and physical activity in preschool children: a cross-sectional study," BMC Public Health, vol. 10, no. 1, p. $733,2010$.

[29] L. J. Griffiths, C. Dezateux, and A. Hill, "Is obesity associated with emotional and behavioural problems in children? Findings from the Millennium Cohort Study," International Journal of Pediatric Obesity, vol. 6, no. 2, pp. E423-E432, 2011.

[30] J. J. Puder and S. Munsch, "Psychological correlates of childhood obesity," International Journal of Obesity, vol. 34, no. S2, pp. S37-S43, 2010.

[31] L. Webber, C. Hill, J. Saxton, C. H. Van Jaarsveld, and J. Wardle, "Eating behaviour and weight in children," International Journal of Obesity, vol. 33, no. 1, pp. 21-28, 2009.

[32] K. A. Copeland, J. C. Khoury, and H. J. Kalkwarf, "Child care center characteristics associated with preschoolers' physical activity," American Journal of Preventive Medicine, vol. 50, no. 4, pp. 470-479, 2016.

[33] M. S. Tremblay, V. Carson, J. P. Chaput et al., "Canadian 24hour movement guidelines for children and youth: an integration of physical activity, sedentary behaviour, and sleep," Applied Physiology, Nutrition, and Metabolism, vol. 41, no. 6, pp. S311-S327, 2016.

[34] R. Iten, M. Lechner, S. Stern, and C. Felfe, Gleichstellung der Geschlechter: welche Rolle spielt die familienergänzende Kinderbetreuung?, 2013.

[35] H. B. G. Ganzeboom, P. M. Degraaf, D. J. Treiman, and J. Deleeuw, "A standard international socioeconomic index of occupational-status," Social Science Research, vol. 21, no. 1, pp. 1-56, 1992.

[36] N. Messerli-Burgy, T. H. Kakebeeke, A. Arhab et al., "The Swiss Preschoolers' health study (SPLASHY): objectives and design of a prospective multi-site cohort study assessing psychological and physiological health in young children," BMC Pediatrics, vol. 16, no. 1, p. 85, 2016.

[37] D. P. Cliff, J. J. Reilly, and A. D. Okely, "Methodological considerations in using accelerometers to assess habitual physical activity in children aged 0-5 years," Journal of Science and Medicine in Sport, vol. 12, no. 5, pp. 557-567, 2009.

[38] N. F. Butte, W. W. Wong, J. S. Lee, A. L. Adolph, M. R. Puyau, and I. F. Zakeri, "Prediction of energy expenditure and physical activity in preschoolers," Medicine \& Science in Sports \& Exercise, vol. 46, no. 6, pp. 1216-1226, 2014.

[39] J. H. Migueles, C. Cadenas-Sanchez, U. Ekelund et al., "Accelerometer data collection and processing criteria to assess physical activity and other outcomes: a systematic review and practical considerations," Sports Medicine, vol. 47, no. 9, pp. 1821-1845, 2017.

[40] WHO Multicentre Growth Reference Study Group, WHO Child Growth Standards: Length/Height-for-Age, Weight-forAge, Weight-for-Length, Weight-for-Height and Body Mass Index-for-Age: Methods and Development, World Health Organization, Geneva, Switzerland, 2006.

[41] T. G. Lohman, A. F. Roche, and R. Martorell, Anthropometric Standardization Reference Manual, Human Kinetics, Champaign, IL, USA, 1988.

[42] S. E. Benjamin, B. Neelon, S. C. Ball, S. I. Bangdiwala, A. S. Ammerman, and D. S. Ward, "Reliability and validity of a nutrition and physical activity environmental selfassessment for child care," International Journal of Behavioral Nutrition and Physical Activity, vol. 4, no. 1, p. 29, 2007.

[43] R. Goodman, "Psychometric properties of the strengths and difficulties questionnaire," Journal of the American Academy of Child and Adolescent Psychiatry, vol. 40, no. 11, pp. 1337-1345, 2001.

[44] F. Petermann, K. Niebank, and H. H. Scheithauer, "Risiken in der frühkindlichen Entwicklung: Entwicklungspsychopathologie der ersten Lebensjahre," in Risiken in der frühkindlichen Entwicklung, p. 384, Hogrefe, Göttingen, Germany, 2000.

[45] J. Wardle, C. A. Guthrie, S. Sanderson, and L. Rapoport, "Development of the children's eating behaviour questionnaire," Journal of Child Psychology and Psychiatry, vol. 42, no. 7, pp. 963-970, 2001.

[46] A. C. Lindsay, K. M. Sussner, J. Kim, and S. Gortmaker, "The role of parents in preventing childhood obesity," The Future of Children, vol. 16, no. 1, pp. 169-186, 2006.

[47] F. E. Harrell, Regression Modeling Strategies: with Applications to Linear Models, Logistic and Ordinal Regression, and Survival Analysis, Springer Series in Statistics, Springer, Cham, Switzerland, 2015.

[48] T. Hastie, R. Tibshirani, and J. Friedman, The Elements of Statistical Learning: Data Mining, Inference, and Prediction, Springer Series in Statistics, Springer, New York, NY, USA, 2009.

[49] A. E. Bauman, R. S. Reis, J. F. Sallis, J. C. Wells, R. J. F. Loos, and B. W. Martin, "Correlates of physical activity: why are some people physically active and others not?," The Lancet, vol. 380, no. 9838, pp. 258-271, 2012.

[50] M. Kuhn, M. Kuhn, and K. Johnson, Applied Predictive Modeling, Springer, New York, NY, USA, 2013.

[51] S. Roberts and G. Nowak, "Stabilizing the lasso against crossvalidation variability," Computational Statistics and Data Analysis, vol. 70, pp. 198-211, 2014.

[52] R. Lockhart, J. Taylor, R. J. Tibshirani, and R. Tibshirani, “A significance test for the lasso," Annals of Statistics, vol. 42, no. 2, pp. 413-468, 2014. 
[53] R. Lockhart, J. Taylor, R. J. Tibshirani, and R. Tibshirani, "Rejoinder: "a significance test for the lasso"," Annals of Statistics, vol. 42, no. 2, pp. 518-531, 2014.

[54] J. Friedman, T. Hastie, and R. Tibshirani, "Regularization paths for generalized linear models via coordinate descent," Journal of Statistical Software, vol. 33, no. 1, pp. 1-22, 2010.

[55] M. Kuhn, S. W. Wing, A. Williams et al., "Caret: classification and regression training. R package version 6.0-73," 2016, http://CRAN.R-project.org/package=caret.

[56] S. van Buuren and K. Groothuis-Oudshoorn, "mice: multivariate imputation by chained equations in R," Journal of Statistical Software, vol. 45, no. 3, pp. 1-67, 2011.

[57] T. Hinkley, J. Salmon, A. D. Okely, K. Hesketh, and D. Crawford, "Correlates of preschool children's physical activity," American Journal of Preventive Medicine, vol. 43, no. 2, pp. 159-167, 2012.

[58] E. A. Schmutz, C. S. Leeger-Aschmann, T. Radtke et al., "Correlates of preschool children's objectively measured physical activity and sedentary behavior: a cross-sectional analysis of the SPLASHY study," International Journal of Behavioral Nutrition and Physical Activity, vol. 14, no. 1, 2017.

[59] J. S. Gubbels, D. H. Van Kann, and M. W. Jansen, "Play equipment, physical activity opportunities, and children's activity levels at childcare," Journal of Environmental and Public Health, vol. 2012, Article ID 326520, 8 pages, 2012.

[60] K. E. Henderson, G. M. Grode, M. L. O'Connell, and M. B. Schwartz, "Environmental factors associated with physical activity in childcare centers," International Journal of Behavioral Nutrition and Physical Activity, vol. 12, no. 1, p. 43, 2015.

[61] A. Bandura, Social Learning Theory, Prentice-Hall Series in Social Learning Theory, Prentice-Hall, Englewood Cliffs, NJ, USA, 1977.

[62] R. J. Parker, E. J. Elliott, A. Georga, and M. Booth, "Developing a charter of physical activity and sport for children and youth," Australian and New Zealand Journal of Public Health, vol. 27, no. 5, pp. 517-519, 2003.

[63] L. M. Vanderloo, P. Tucker, A. M. Johnson, M. M. van Zandvoort, S. M. Burke, and J. D. Irwin, "The influence of centre-based childcare on preschoolers' physical activity levels: a cross-sectional study," International Journal of Environmental Research and Public Health, vol. 11, no. 2, pp. 1794-1802, 2014.

[64] P. J. Collings, S. Brage, C. L. Ridgway et al., "Physical activity intensity, sedentary time, and body composition in preschoolers," American Journal of Clinical Nutrition, vol. 97, no. 5, pp. 1020-1028, 2013.

[65] M. Golan, "Parents as agents of change in childhood obesity-from research to practice," International Journal of Pediatric Obesity, vol. 1, no. 2, pp. 66-76, 2006.

[66] H. G. Lawman and D. K. Wilson, "A review of family and environmental correlates of health behaviors in high-risk youth," Obesity, vol. 20, no. 6, pp. 1142-1157, 2012.

[67] V. Carson, E. Y. Lee, L. Hewitt et al., "Systematic review of the relationships between physical activity and health indicators in the early years (0-4 years)," BMC Public Health, vol. 17, no. 1, p. 854, 2017. 


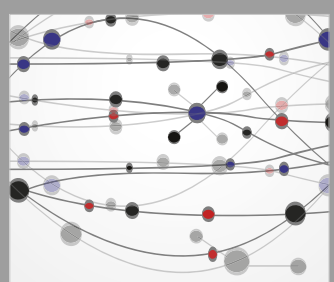

The Scientific World Journal
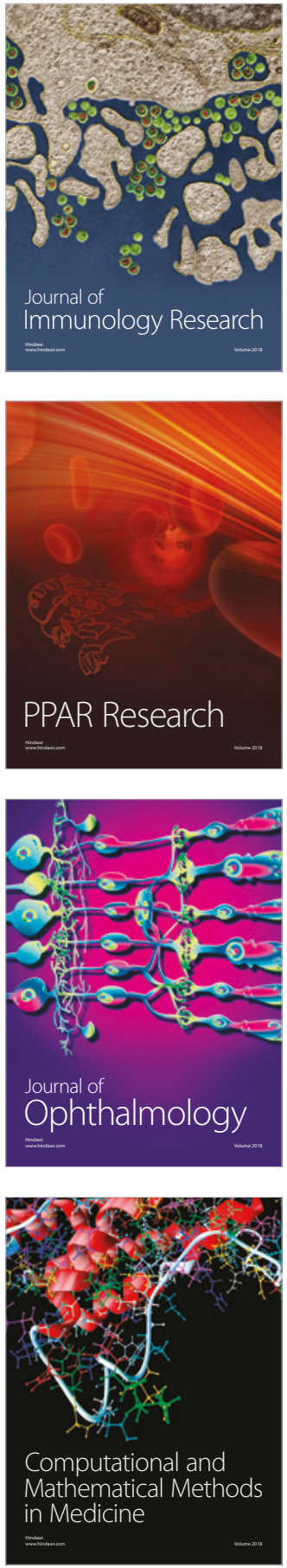

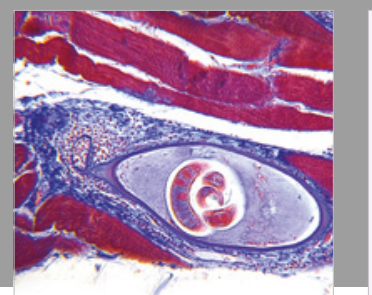

Gastroenterology Research and Practice

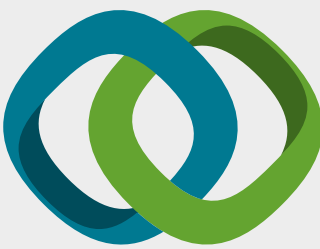

\section{Hindawi}

Submit your manuscripts at

www.hindawi.com
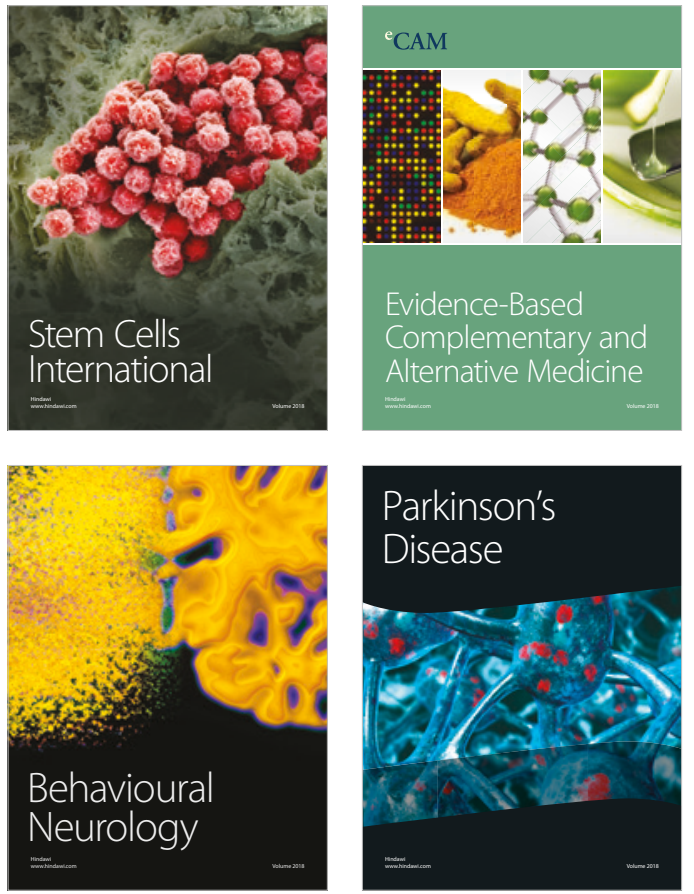

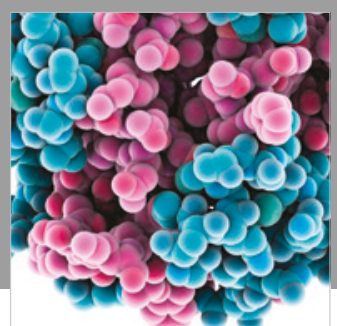

ournal of

Diabetes Research

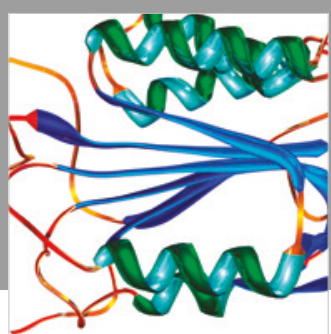

Disease Markers
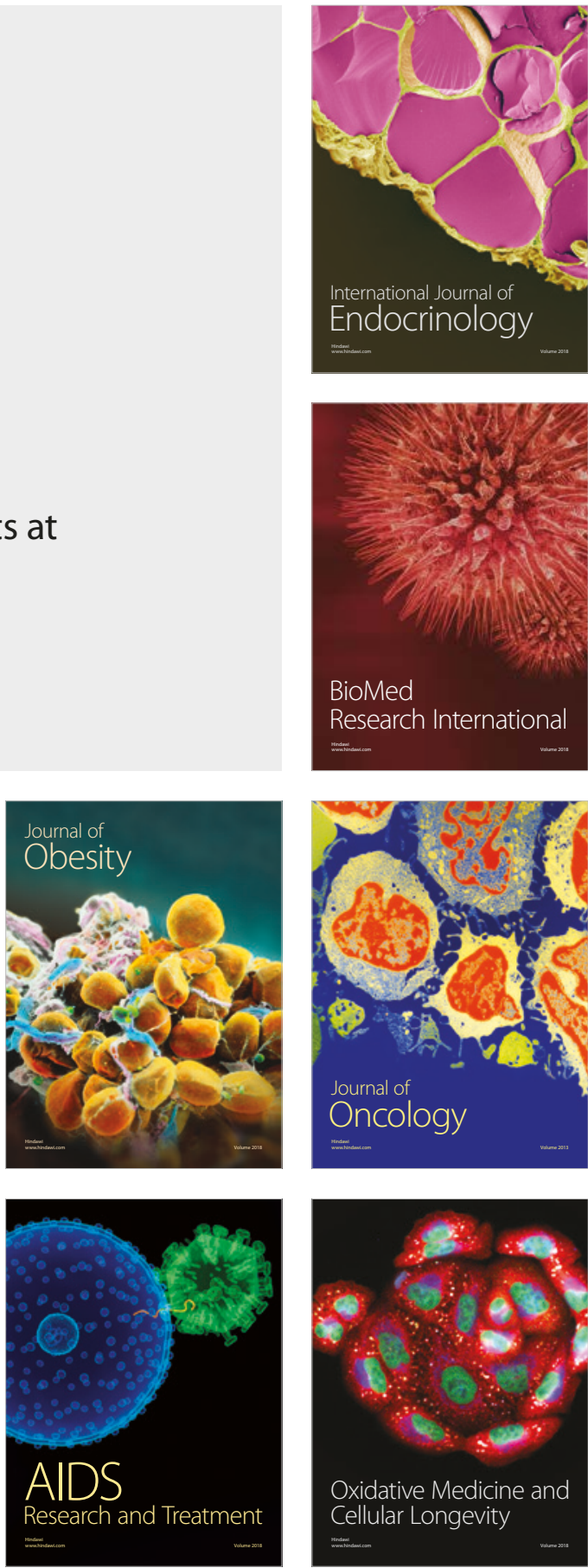